Revista de Investigación Educativa 24

enero-junio, 2017 | ISSN 1870-5308 | Xalapa, Veracruz

Instituto de Investigaciones en Educación | Universidad Veracruzana

Fortalecimiento de las competencias docentes para atender el cyberbullying en una secundaria pública colombiana

Strengthening teaching competence for addressing cyberbullying in a Colombian public junior high school

Mtro. Mauricio Alejandro Castro-Granados Maestro de secundaria y media técnica Colegio Acción Comunal de Cundinamarca, Colombia macgranados@gmail.com

Mtra. Carmen Medina-Almeida

Profesora de posgrado

Tecnológico de Monterrey, México

car.almeida@itesm.mx

Dr. Leonardo David Glasserman Morales

Profesor-Investigador, Director del Programa de Maestría en Administración de Instituciones Educativas

Tecnológico de Monterrey, México glasserman@itesm.mx 
El objetivo general del estudio fue analizar las competencias docentes que se pueden fortalecer para la atención de los casos de cyberbullying que se presentan entre los estudiantes de una secundaria pública colombiana. La metodología comprendió un estudio mixto y la muestra no probabilística estuvo conformada por 21 docentes y 62 estudiantes. En una primera etapa cualitativa, se entrevistó al profesorado y a la coordinadora de convivencia, en su calidad de directiva. Además, se diseñó e impartió un curso de capacitación docente en Moodle sobre los aspectos fundamentales para el manejo del cyberbullying. Posterior al curso (etapa cuantitativa), se aplicaron encuestas a docentes y estudiantes. Finalmente, se concluyó que el profesorado requiere fortalecer las competencias comunicativas, interpersonales, el uso de TIC y la resolución de conflictos, para generar estrategias que permitan atender y prevenir el cyberbullying. Dichas acciones deben involucrar a padres de familia y directivos del colegio.

Palabras claves: Competencias docentes; Moodle; cyberbullying; secundaria.

The general objective of the study was to analyze the teaching skills that can be strengthened for handling cyberbullying cases, occurring among students of a Colombian public school. The methodology comprised a mixed study and a non-probabilistic sample consisted of 21 teachers and 62 students. In the first qualitative stage, the teachers and the coexistence coordinator (directive) were interviewed. In addition, it was designed and implemented a teacher training course in Moodle on key aspects for the management of cyberbullying. After the course (quantitative phase), surveys were applied to teachers and students. Finally, it was concluded that teachers need to strengthen communication skills, interpersonal skills, use of ICт and conflict resolution, in order to generate strategies to handle and prevent cyberbullying. Those actions must involve parents and school managers.

Keywords: Teaching competences; Moodle; cyberbullying; junior high-school. 
Fortalecimiento de las competencias docentes para atender

el cyberbullying en una secundaria pública colombiana

Mauricio Alejandro Castro-Granados, Carmen Medina-

Almeida, Leonardo David Glasserman Morales

\title{
Fortalecimiento de las competencias docentes para atender el cyberbullying en una secundaria pública colombiana ${ }^{1}$
}

\author{
Strengthening teaching competence for addressing \\ cyberbullying in a Colombian public junior high school
}

\section{Introducción}

T a docencia es una vocación y los maestros son la principal fuerza a favor de la equiLdad, el acceso y la calidad de la enseñanza (Organización de las Naciones Unidas para la Educación, la Ciencia y la Cultura [UNESCO], 2013). El docente en la sociedad del conocimiento debe asumir múltiples desafíos, ejemplo de ello es cuestionarse sobre su práctica pedagógica a partir de la reflexión, investigación y análisis del proceso de aprendizaje de los educandos. Por lo tanto, es necesario indagar sobre las competencias docentes a fin de transformar la cultura escolar, según las demandas sociales y laborales. Todo ello, orientado hacia una formación integral del alumnado.

Asimismo, el ambiente desarrollado al interior de las escuelas permite ver una problemática disciplinaria y académica, en gran parte ocasionada por la desmotivación de los educandos ante el modelo tradicional, siendo éste uno de los principales obstáculos en el ejercicio docente y otro de los elementos generadores del conflicto escolar al interior del aula (Vaello, 2003). Este fenómeno ha trascendido al contexto virtual, a partir de la incorporación de apoyos tecnológicos y el uso de redes sociales en el ámbito educativo, en particular por parte de los aprendices, donde constantemente se presentan conductas inapropiadas $u$ ofensivas que afectan emocionalmente a sus pares. Este tipo de maltrato social y virtual sólo requiere de un medio de comunicación digital para poder realizarse en cualquiera de sus modalidades (Kowalski, Limber \& Agatston, 2010). Lo anterior se conoce como cyberbullying.

1. Esta investigación fue aceptada para presentarse en el Primer Congreso Internacional de Innovación Educativa realizado en 2014 en el Campus Ciudad de México del Tecnológico de Monterrey. Sin embargo, la ponencia no se llevó a cabo en virtud de que el coautor asignado tuvo un problema de salud y la investigación no fue incluida en las memorias del evento. 
Lo expuesto líneas arriba se agravó en una institución secundaria colombiana, donde específicamente se presentaron situaciones como el desinterés en el estudio por parte de los alumnos, la discriminación de compañeros, el dominio que quieren ejercer algunos estudiantes dentro del aula, la falta de atención del docente y también de los padres ante los problemas. Estas consideraciones generaron un deterioro de los valores en los alumnos y su consecuente apatía hacia el aprendizaje, situación que también afectó los procesos académicos en la institución educativa y permitió el aumento de los conflictos escolares virtuales, además de la pasividad de los maestros para atender los casos de cyberbullying, aunado a la falta de capacitación docente.

En consecuencia, el presente estudio se basó en la pregunta general de investigación: ¿cuáles son las competencias docentes que se pueden fortalecer para la atención del cyberbullying que se presenta entre los estudiantes de una secundaria colombiana? Como objetivos específicos se establecieron: 1) conocer las estrategias que implementa el profesorado para atender, manejar y prevenir el cyberbullying en la secundaria que fue escenario educativo de la indagación; 2) determinar las competencias que necesitan fortalecer los docentes para manejar y prevenir el cyberbullying en secundaria; 3) analizar el rol asumido por los profesores ante los conflictos escolares virtuales que se presentan entre los estudiantes; 4) conocer la actitud del profesorado respecto al uso de Moodle, como apoyo en su capacitación docente para el manejo del cyberbullying, y 5) determinar la responsabilidad de la institución educativa en cuanto a la necesidad de brindar capacitación docente para prevenir y atender esa clase de conflictos.

\section{Marco teórico}

\subsection{Formación basada en competencias: competencias docentes}

La actual sociedad se encuentra en constante transformación, por lo que los sujetos se deben adaptar de forma eficaz y rápida a dichos cambios. En respuesta a ello, surge la Formación Basada en Competencias ( $\mathrm{FBC}$ ) considerada por algunos autores como enfoque o modelo educativo. No obstante, es una alternativa para solucionar diversas necesidades del ámbito personal, social, profesional, cultural y educacional. En palabras de García y Martínez (2014), la FBC es la formación integral del estudiante, que incluye una serie de habilidades y capacidades acordes a las diferentes situaciones en las que éste se desenvuelve. 
La FBC busca la evolución o reingeniería de la educación tradicional, brindando al proceso de enseñanza un viraje que permite fortalecer el aprendizaje (Villa \& Poblete, 2007). Es interesante observar que la FBC permite al estudiante ser el centro del proceso de enseñanza aprendizaje; el docente es un motivador y mediador que está al nivel del educando para dinamizar las relaciones y promover estrategias para el aprendizaje, sin necesidad de ser el actor de mayor relevancia. Lo anterior se confirma en la investigación de Jiménez, González y Hernández (2011), donde se manifiesta que la FBC es un trabajo cooperativo entre las partes a partir de un equilibrio en el desarrollo de conocimientos, actitudes, habilidades y valores.

Este enfoque aborda las competencias genéricas en comparación con las específicas del sistema tradicional, otorgando una ventaja en la formación de un profesional de alto desempeño. Es por ello que dicha formación facilita un aprendizaje complejo, que permite incorporar el saber conocer, el saber hacer y el saber ser (Tobón, 2005). En opinión de Echeverría (2002), estos tres saberes conforman las competencias.

De igual manera, la FBC permite a los estudiantes, maestros y profesionales ser capaces de indagar, buscar y seleccionar la información, con el fin de resolver situaciones o problemas reales del entorno. Esto permite la aportación de un conocimiento compuesto para enfrentar la complejidad de las diferentes situaciones que se pueden presentar en el transcurso de la vida, puesto que se omite el aprendizaje fragmentado en asignaturas o temáticas de los saberes. En consecuencia, se busca la formación integral del sujeto (Cano, 2005). Finalmente, puede señalarse que la FBC se contempla como una alternativa a la educación tradicional, en virtud de los diferentes cambios presentados en la sociedad, especialmente en el ámbito social, económico y tecnológico. Lo anterior se traduce en el desarrollo de capacidades y habilidades que permiten la constante comunicación y el aprendizaje cooperativo, desarrollando nuevas habilidades y competencias laborares en aprendices y docentes (Navés, 2015).

\subsection{Cyberbullying: características y repercusiones psicológicas en el alumno de secundaria}

Todo conflicto implica relaciones fragmentadas, donde no ha sido posible establecer la armonía o mutua aceptación entre las personas que integran cierto grupo social (Puig, 1997). Estas situaciones de discordia se inician cuando un sujeto o grupo, experimentan una sensación de disgusto y enfrentamiento con otra persona o grupo social. Puede concluirse, a modo de síntesis, que un conflicto supone una oposición 
de intereses sobre un mismo tema, o cuando el mutuo acuerdo de las distintas partes no puede alcanzarse (Grasa, 1991; Oyhanarte, 1996).

A partir de las argumentaciones de Hernández (2004); García, Joffre, Martínez y Llanes (2011), el conflicto escolar se puede catalogar como un proceso que vulnera la integridad física, social y/o psicológica de otra persona o grupo de individuos, dentro o fuera del ambiente educativo, caracterizándose por una situación de discordia o enfrentamiento entre personas, o incluso entre un individuo y un determinado grupo social. En el ámbito educacional, esta situación está presente en la vida de los alumnos, ya sea de forma directa o indirecta, e implica situaciones de pugna entre dos o más individuos, pudiendo comprender objetivos, intereses, valores, conductas o fines contrapuestos, donde siempre hay un inicio, un desarrollo y un desenlace que determina el tipo de interacción y confrontación que se puede presentar.

Conviene tener presente que el conflicto aborda dos dimensiones: la positiva y la negativa. En cuanto a la primera, el conflicto puede ser positivo pues brinda la posibilidad de generar aprendizaje, a partir de la reflexión sobre las acciones emprendidas para solucionar o poner fin al conflicto y, por ende, eso también genera crecimiento personal. La dimensión negativa se asocia con la violencia (física o psicológica) presentada entre las partes (Moore, 1995; Pantoja, 2005; Vaello, 2003). De igual manera, el conflicto vincula componentes de diversa naturaleza, razón por la cual todos los esfuerzos deben dirigirse a estos elementos para orientar la intervención por parte del docente. Por su parte, De la Caba y López (2013), consideran que el componente positivo son las acciones encaminadas a dialogar con el agresor y solicitar que el acoso finalice. Mientras que las acciones negativas o pasivas están relacionadas con no hacer nada o guardar silencio.

Al respecto, es necesario considerar los resultados de las investigaciones realizadas por Aznar, Cáceres e Hinojo (2007), Lukas y Santiago. (2004), al igual que los obtenidos en la Encuesta Nacional de Deserción Escolar (ENDE), aplicada por el Ministerio de Educación Nacional (2009). En ellas se identificaron los siguientes problemas: la conducta inapropiada en el aula, el maltrato entre iguales y el cyberbullying. Este último conflicto es de carácter emergente debido a que combina la agresión entre sujetos bajo la misma condición e incorpora el uso de las Tecnologías de la Información y Comunicación (TIC).

No obstante, es necesario precisar que las TIC se idearon para fortalecer y mejorar los procesos a nivel empresarial, escolar y social. En palabras de Henríquez-Ritchie y Organista (2010), el uso de la tecnología computacional abarca dos dimensiones: la 
académica y la no académica. La última hace referencia al manejo de redes sociales, búsqueda de información y uso de aplicaciones recreativas. Sin embargo, éstas son utilizadas por algunos individuos como escenario de distintas formas de acoso, violencia u hostigamiento entre iguales (Hernández, 2004). Por lo tanto, el cyberbullying se puede entender como el desarrollo de actitudes, acciones o comportamientos agresivos, mal intencionados e insultantes entre iguales, a través de la comunicación e interacción que se genera al usar medios de comunicación electrónicos; todas ellas, conductas prolongadas a través del tiempo y que también constituyen elementos distintivos del acoso virtual o cyberbullying (Giménez, 2015; Luengo, 2011; García et al., 2011).

Una de las características fundamentales de este tipo de conflicto en las instituciones educativas radica en que la sociedad actualmente está permeada por los adelantos tecnológicos, lo que determina las expectativas, necesidades, inquietudes e intereses de los jóvenes. Asimismo, este conflicto involucra especialmente a los adolescentes que oscilan entre los 10 y 16 años, que están creciendo y madurando, razón por la cual suelen ser obstinados, características detonadoras del acoso virtual (Luengo, 2011). Además, se considera que las consecuencias de las víctimas se pueden catalogar en dos tiempos. En primera medida, a corto y mediano plazo se presentan repercusiones como estrés, humillación, ansiedad, ira, impotencia, fatiga, pérdida de confianza en sí mismo, ocasionando secuelas psicológicas y físicas. En segunda instancia, se considera el acoso por tiempo prolongado, los individuos tienden a desarrollar cambios de personalidad y características de estrés postraumático, lo cual implica que disminuirá la capacidad de la persona de relacionarse e interactuar adecuadamente con otros individuos (Olweus, 2001).

Por lo tanto, a partir de las apreciaciones de Luengo (2011) y Hernández (2004), al igual que las investigaciones realizadas por Kowalski (2010), el Instituto Nacional de Tecnología de la Comunicación (INTECO, 2009), Aftab (2006), Blaya (2010) y Garaigordobil (2011) sobre el tema de estudio, se puede afirmar que el cyberbullying es una conducta que tiende a masificarse según las habilidades en el uso y manejo de las TIC, especialmente porque los jóvenes no reflexionan ni miden el alcance de esta conducta inapropiada y muchas veces ilícita. Aunado a esto, el rol del agresor, la víctima pasiva, la víctima provocadora y el observador, pueden modificarse o alternarse rápidamente, pues en un escenario digital, cualquier persona (sólo para hacer demostración de supremacía) puede generar agresiones consideradas como cyberbullying. Según la investigación de Prieto, Carrillo y Lucio (2015), una de las acciones más recurrentes en este tipo de acoso es la de ridiculizar a los compañeros a partir de acciones tales como la publicación de fotografías o videos. 
Fortalecimiento de las competencias docentes para atender

el cyberbullying en una secundaria pública colombiana

Mauricio Alejandro Castro-Granados, Carmen MedinaAlmeida, Leonardo David Glasserman Morales

Por último, el silencio es una consecuencia de este conflicto, motivo por el cual los padres de familia y los docentes desconocen si sus hijos han sido o están siendo víctimas de esta problemática. Tal como refiere Puig (1997), el beneficio que puede generarse con la intervención del docente ante los conflictos de cyberbullying es evitar daños físicos y emocionales excesivos e innecesarios; todo dependerá de la pronta reacción que emprendan los maestros y los padres de familia.

En virtud de lo anterior, una de las repercusiones más recurrentes entre los jóvenes de secundaria es la falta de aceptación entre sus pares, la cual genera baja autoestima, soledad, aislamiento social y ausentismo escolar (Cowie, 2013), y en consecuencia, el rendimiento académico se ve afectado (Lucas, Pérez de Albéniz \& Giménez, 2016), provocando ansiedad y situaciones de indisciplina. Además, el otro elemento a considerar es el auto aislamiento de la víctima, pues pierde la capacidad de relacionarse con los demás por temor a ser violentado y, en casos extremos, se puede presentar el suicidio, o enfermedades psicosomáticas (Blaya, 2010; Olweus, 2001; Piñuel, 2002). Asimismo, este problema se asocia con estrés emocional, ansiedad social, consumo de sustancias, síntomas depresivos y, con el paso del tiempo, se presentan pensamientos suicidas e intentos de suicidio (Borges, Bottino, Gómez, Lobo \& Silva, 2015).

\subsection{Rol del docente frente al cyberbullying}

El cyberbullying como problemática al interior de las instituciones educativas de nivel secundaria, implica considerar factores como la comunicación, interacción y el uso de las TIC en los procesos de enseñanza-aprendizaje. Las investigaciones de Aftab (2006) y Kowalski, Limbert y Agatston (2010), al igual que las leyes o programas internacionales como la Ley 1620 del 15 de marzo de 2013 en Colombia (Ley de Infancia y Adolescencia) (Gobierno Nacional de Colombia, 2013), la Guía legal sobre cyberbullying y grooming (INTECO, 2009) y la Guía de recursos para centros educativos en casos de ciberacoso (Luengo, 2011), enuncian que el principal factor para evitar esta situación es la responsabilidad de los padres de familia y de las instituciones educativas. Por lo tanto, se puede afirmar que esta problemática involucra a todos los responsables del proceso educativo: directivos, personal administrativo, docentes y padres de familia. Es por ello que se requiere el compromiso de toda la comunidad en este tipo de conflicto, para diseñar y establecer estrategias de intervención y prevención adecuada, a fin de poder brindar una formación integral de los estudiantes (Morales, Serrano, Miranda \& Santos, 2014). 
$\mathrm{Al}$ respecto, Torres (2010) considera que el rol del docente ante el conflicto es una responsabilidad y compromiso de todos los maestros de la institución, con el fin de asegurar en el estudiante el desarrollo de su sensibilidad moral, la formación en valores, la toma y participación de decisiones éticas y el desarrollo de la autonomía. Asimismo, Puig (1997) considera que la labor central del docente es ser mediador: debe reconocer, aceptar y comentar las diferentes dificultades o adversidades que se pueden presentar en un problema. Es por ello que el conciliador debe desarrollar competencias personales e interpersonales que le permitan escuchar, entender, identificar, analizar el conflicto con el fin de poder construir una solución concertada entre la víctima y el agresor.

Torres (2010) manifiesta que el actor que interactúa directamente con el estudiante es el docente, por lo que es necesario fortalecer la competencia docente para atender los conflictos escolares. Para este fin, el docente tiene que asumir uno de muchos retos que se presentan en su ejercicio profesional. En otras palabras, el profesor debe saber identificar las necesidades de cambio (flexibilidad de pensamiento), actualizarse permanentemente, establecer relaciones de colaboración y de trabajo común con los maestros de su institución, enfrentar los problemas y asumir el compromiso de superarlos proactivamente. Los docentes también deberán incluir en su desempeño el uso de las TIC (Navés, 2015), ya que permiten realizar ajustes en la práctica docente, dinamizando el proceso de aprendizaje del estudiante y propiciando una nueva oportunidad de conocimiento en la docencia.

De igual manera, Frade (2009) afirma que es necesario diseñar y desarrollar situaciones o escenarios de aprendizaje interesantes y atractivos, en los que el alumno desarrolle sus propias competencias al resolver el conflicto cognitivo que le presentan, ya sea por la aplicación de estrategias pedagógicas o al involucrar elementos tecnológicos, identificando las oportunidades de aprendizaje. En ese sentido, el docente también debe reconocer los efectos negativos que se pueden generar por el uso inadecuado de los apoyos tecnológicos, así como promover estrategias para prevenir esas conductas.

Por su parte, Borges et al. (2015), afirman que los padres de familia y los maestros deben conocer las implicaciones y riesgos de las comunicaciones electrónicas y, por ende, facilitar el diálogo sobre el tema, con el fin de brindar al estudiante una comunicación efectiva de los diferentes sucesos que se presentan. Por todo lo anterior, es pertinente enfatizar que en el escenario educativo considerado en el estudio sobresalió la importancia de iniciar la capacitación docente, a fin de profesionalizar a los maestros de la institución en la atención del cyberbullying. Como parte del proyecto, también se 
diseñó e impartió un curso en Moodle, situación que no se había ofrecido antes para los docentes. Obviamente, se partió de la importancia de ofrecer una primera capacitación lo más cercana al perfil e intereses del profesorado.

\section{Método}

La investigación consideró un enfoque mixto que se caracterizó por la recolección, análisis e integración de datos cualitativos y cuantitativos para realizar metainferencias producto de todos los datos recolectados y con el objetivo de lograr un mayor entendimiento del fenómeno indagado (Johnson \& Onwuegbuzie, 2004). En consecuencia, se obtuvieron variados datos por la multiplicidad de técnicas de recolección, lo cual mejoró la indagación y análisis de los mismos (Todd, Nerlich \& McKeown, 2004).

Por lo anterior, se implementó un diseño mixto de tipo exploratorio secuencial y en modalidad comparativa (DEXPLOS), tal como lo establecen Hernández, Fernández y Baptista (2010), tomando en cuenta la pregunta general de investigación y los objetivos formulados en el planteamiento del problema.

Es necesario aclarar que esta modalidad del diseño no está conformada por fases independientes. Por el contrario, se realizó un proceso comparativo entre ambas fases para poder establecer una discusión final del estudio y realizar metainferencias que permitieron responder las preguntas de investigación (Hernández et al., 2010; Teddlie \& Tashakkori, 2009). Se inició con la recolección de los datos cualitativos y después se obtuvieron los cuantitativos. Esos últimos facilitaron la interpretación de los resultados cualitativos (Hernández et al., 2010). De igual manera, se pudieron explorar distintos niveles del problema de estudio y se obtuvo una mayor variedad de perspectivas; según Johnson y Onwuegbuzie (2004), eso se denomina riqueza interpretativa.

La muestra no probabilística (Hernández et al., 2010) estuvo constituida por los 21 maestros del turno matutino de la sede principal de la institución, ubicada en el Departamento de Cundinamarca, en Colombia (donde labora el primer autor). Específicamente, colaboraron en el estudio: 15 mujeres (71\%) y 6 varones (29\%). Su participación fue voluntaria; dichos docentes también eran titulares de grupos de alumnos de secundaria y tenían a su cargo diferentes áreas del conocimiento del centro educativo. Las edades de los profesores participantes oscilaron entre los 30 y 60 años de edad y algunos tenían una experiencia laboral superior a los 20 años. De igual manera, la mayoría del equipo docente de la institución educativa, contaba con un grado de es- 
tudios de posgrado equivalente a especialización. Por otra parte, también participó la coordinadora de convivencia (como directiva), debido a que el manejo de conflictos era dirigido por ella a nivel institucional. La coordinadora había laborado durante 25 años en la institución, al momento de realizarse el estudio.

También se contó con la participación de 62 estudiantes de educación media técnica (equivalente a secundaria en México), pertenecientes al turno matutino; 38 de ellos integraron el Grupo 1001 con énfasis en redes de computadores y los 24 restantes fueron del Grupo 1102, relativo a la formación en gestión empresarial. El total de la muestra del alumnado estuvo conformado por 21 varones (34\%) y 41 mujeres (66\%), cuyas edades oscilaron entre los 15 y 17 años.

Sobre la metodología, se contemplaron instrumentos en cada fase, siendo primero la etapa cualitativa y, posteriormente, la cuantitativa (Teddlie \& Tashakkori, 2009). En relación con los instrumentos aplicados, en la fase cualitativa se aplicó una entrevista semiestructurada a cinco de los 21 docentes que integraron la muestra y que aceptaron ser entrevistados. Dicho instrumento incluyó 16 preguntas abiertas, con las cuales se pretendió conocer la perspectiva del docente sobre el conflicto escolar virtual. Las preguntas abordaron aspectos como el ambiente escolar y las repercusiones que tiene el cyberbullying en alumnos de secundaria, además de su conocimiento acerca de esta problemática, haciendo énfasis en causas, casos, consecuencias y estrategias que podría implementar el profesorado (o que ya está poniendo en práctica), ante una situación de esta índole. También se abordaron estrategias de intervención, mediación y prevención, desde el punto de vista de su rol docente. Además, se consideraron cuestiones relacionadas con las competencias docentes requeridas para manejar esta situación; el interés de ser capacitados para mediar y atender el problema, al igual que los desafíos que deben afrontar los profesores ante este conflicto escolar, así como la intervención institucional.

En esta primera fase cualitativa también se aplicó otra guía de entrevista semiestructurada a la coordinadora de convivencia, en su calidad de directiva. La guía constó de 12 preguntas abiertas y se pretendió conocer su perspectiva respecto a los casos que se han presentado sobre cyberbullying, es decir, cómo afrontan los docentes esta clase de conflicto (desde la visión de la coordinadora), las estrategias institucionales aplicadas, además de la trascendencia de la responsabilidad del colegio en esta problemática y aspectos sobre capacitación docente para mediar y prevenir el cyberbullying. Este instrumento se aplicó a esa directiva, pues ella manejaba los conflictos de acoso virtual en la institución. 
Posterior a la fase cualitativa, se diseñó e impartió el curso de capacitación docente en Moodle, actividad que nunca se había desarrollado en la institución educativa. Después, en la fase cuantitativa se aplicaron dos encuestas. Es preciso aclarar que los instrumentos de esta etapa sólo se aplicaron después de concluido el curso de capacitación y con los docentes que decidieron participar en la indagación. Las dos encuestas se diseñaron con escalamiento Likert (Hernández et al., 2010) y se aplicaron a maestros y estudiantes.

Al respecto, la primera encuesta se aplicó al total de profesores que integraron la muestra; este instrumento versó sobre la capacitación docente para la atención del cyberbullying, impartido a través de Moodle. El instrumento contempló 15 preguntas cerradas, las cuales estaban orientadas a conocer la opinión de los docentes, respecto al proceso de capacitación recibido para la atención del cyberbullying, es decir, su experiencia de aprendizaje. También se les cuestionó sobre cuál era el rol que debían asumir ante esta problemática, considerando que son profesionales de la educación.

La segunda encuesta se aplicó al total de estudiantes y constó de 23 preguntas cerradas. La finalidad del instrumento fue conocer la actitud de los educandos en temas como el cyberbullying en la institución: si lo habían padecido, si sabían cómo reaccionar ante esta problemática y a quién podían recurrir en caso de padecer cyberbullying. Asimismo, se incluyeron ítems vinculados a su opinión sobre el apoyo recibido por parte del profesorado en dicha problemática y respecto a sus competencias docentes para la atención del cyberbullying. Para tal fin, las preguntas contemplaron cinco alternativas de respuesta: totalmente de acuerdo (1); de acuerdo (2); ni de acuerdo ni en desacuerdo (3); en desacuerdo (4); totalmente en desacuerdo (5).

Cabe mencionar que para la selección de la coordinadora, los maestros y los estudiantes que integraron la muestra, se tomó en cuenta el comentario del director del colegio y se estableció su participación voluntaria en la indagación. Asimismo, la aplicación de las entrevistas fue realizada directamente por el investigador, con el fin de identificar las diferentes formas de expresión de los participantes (Creswell, 2009). Se utilizó una cámara de video con el fin de poder recurrir a la información en cualquier momento, y principalmente, porque facilita la transcripción de la entrevista en su totalidad (Valenzuela \& Flores, 2012).

Por otro lado, las encuestas mediante los dos cuestionarios autoadministrados tuvieron dos momentos de aplicación; primero se aplicó a los docentes al finalizar la sesión plenaria del curso de Moodle. Después se realizó la segunda encuesta con los 
Fortalecimiento de las competencias docentes para atender

el cyberbullying en una secundaria pública colombiana

Mauricio Alejandro Castro-Granados, Carmen MedinaAlmeida, Leonardo David Glasserman Morales

estudiantes durante la clase de Tecnología e Informática, con el fin de garantizar la interacción entre los aprendices y el maestro titular. De esa manera se recolectaron los datos-dentro del ambiente de clase.

En virtud de que el estudio fue mixto y se implementó un diseño DEXPLos (Hernández et al., 2010), fue necesario realizar el análisis de los datos cualitativos y cuantitativos por separado. Los datos cualitativos fueron codificados y categorizados para un análisis taxonómico (Valenzuela \& Flores, 2012). Los datos cuantitativos se sometieron a un análisis de estadística descriptiva con medidas de tendencia central y de dispersión (distribuciones de frecuencias absolutas y relativas), previa codificación y uso del libro de códigos para ambas encuestas. Todo ello, permitió realizar una posterior discusión entre los métodos y fases del estudio, mediante la triangulación final con los fundamentos teóricos (Hernández et al., 2010; Teddlie \& Tashakkori, 2009). De esa forma se pudo responder la pregunta principal del estudio y cumplimentar los objetivos formulados en el planteamiento del problema.

\section{Presentación y análisis de resultados}

En este apartado se abordan los datos más significativos recolectados en cada una de las fases de este estudio. Primero, se realiza la presentación de resultados según las etapas de la indagación y los instrumentos aplicados. Posteriormente, se discuten y triangulan con los fundamentos teóricos, para responder la pregunta general de investigación.

\subsection{Presentación de resultados}

Después de la aplicación de las entrevistas (fase cualitativa-primera etapa), cada una de ellas se transcribió en su totalidad. Posteriormente, se realizó la codificación y categorización, según las recomendaciones brindadas por Hernández et al. (2010) y Valenzuela y Flores (2012). Para ello, se seleccionaron segmentos de contenido, unidades de análisis o respuestas, temas, patrones y se establecieron categorías que agruparon las mismas. En esta etapa, también fue prioritario el uso del diario de campo y la bitácora de análisis (Hernández et al., 2010; Merriam, 2009).

Tomando en cuenta las preguntas de investigación y los constructos de este estudio, se consideraron segmentos de contenido, identificándose unidades de datos o de 
análisis. También se tomaron en cuenta los más sobresalientes y finalmente se establecieron temas, patrones y categorías para las entrevistas de los docentes (Tabla 1) y para la entrevista con la coordinadora, respectivamente (Tabla 2).

\section{Tabla 1. Categorías emergentes-Repuestas a la entrevista semiestructurada por los docentes}

\begin{tabular}{l} 
Categoría (Código) \\
\hline Perspectiva sobre el cyberbullying $(P C B)$ \\
\hline Causas del cyberbullying (CACB) \\
\hline Incremento del cyberbullying (ICB) \\
\hline Consecuencias del cyberbullying (COCB) \\
\hline Casos de cyberbullying (CVCB) \\
Conocimiento sobre el manejo institucional del cyberbullying (MICB) \\
\hline Rol docente para la atención, mediación y prevención del cyberbullying (RD-AMP-CB) \\
\hline Competencias docentes para la atención, mediación y prevención del cyberbullying (CD-AMP-CB) \\
\hline Responsabilidad de la comunidad educativa frente al cyberbullying (RCE-CB) \\
\hline Responsabilidad institucional frente al cyberbullying(RICB) \\
\hline
\end{tabular}

Fuente: Elaboración propia.

\section{Tabla 2. Categorías emergentes-Repuestas a la entrevista semiestructurada con la coordinadora de convivencia}

\begin{tabular}{l}
\hline \multicolumn{1}{c}{ Categoría (Código) } \\
\hline Perspectiva sobre el cyberbullying $(P C B)$ \\
\hline Causas del cyberbullying (CACB) \\
\hline Consecuencias del cyberbullying (COCB) \\
\hline Manejo institucional del cyberbullying (MICB) \\
\hline Estrategias institucionales para la atención, mediación y prevención del cyberbullying (EI-AMP-CB) \\
\hline Rol docente para atender, mediar y prevenir el cyberbullying (RD-AMP-CB) \\
\hline Responsabilidad institucional frente al problema del cyberbullying $(R I C B)$ \\
\hline
\end{tabular}

Fuente: Elaboración propia. 
Particularmente, el análisis de contenido evidenció lo siguiente: los docentes emitieron respuestas muy precisas sobre cómo conceptualizaban el cyberbullying. De igual manera, consideraron tres causas que influyen para la presencia de este tipo de conflictos: las condiciones sociales, las relaciones interpersonales y el auge de las redes sociales. Además, la mayoría de los maestros reconoció que no estaba capacitada para atender un caso de cyberbullying, pues al momento del estudio no sabía utilizar las TIC y desconocía aspectos psicológicos relacionados con ese conflicto, como sus consecuencias frecuentes y el apoyo emocional que se debe brindar al estudiante que lo padece. Asimismo, los docentes consideraron que sería conveniente fortalecer las competencias orientadas a la resolución de conflictos, el uso adecuado de las TIC, las competencias comunicativas, éticas, psicológicas, interpersonales y pedagógicas para poder atender, mediar y prevenir el cyberbullying (Tabla 1).

Por su parte, la coordinadora de convivencia de la institución educativa manifestó que la problemática del cyberbullying es una constante entre los jóvenes y ellos desconocen las implicaciones que involucra este tipo de agresión. Además, la directiva argumentó que los docentes no están preparados para atender, mediar y prevenir el cyberbullying y que actualmente el colegio carece de un método definido para poder manejar esta clase de conflicto (Tabla 2).

Sobre la fase cuantitativa (segunda etapa), tal como se indicó antes, se aplicaron dos encuestas: la primera dirigida a los docentes de la institución y la segunda, a los estudiantes de grupos de media técnica (Grupos 1001 y 1102) en la cual se habían presentado agresiones de cyberbullying. El análisis de los datos cuantitativos inició con la codificación de los datos recolectados, a través de sus respectivos instrumentos, tal como lo establecen Hernández et al. (2010), para ello se elaboraron los libros de códigos en Excel de cada una de las encuestas. Con esos libros o documentos de códigos se tuvo una localización de las variables mediante códigos (valores numéricos) asignados para sus categorías, ítems o preguntas y alternativas de respuestas, lo que también se vinculó con una matriz o base de datos en Excel. Todo ello, para realizar un análisis de estadística descriptiva de cada una de las encuestas aplicadas (Hernández et al., 2010), donde se consideraron medidas de tendencia central y de dispersión, así como la presentación de los datos a través de distribuciones de frecuencias absolutas y relativas. Lo anterior también sustentó la coherencia de los resultados producidos por los instrumentos. Finalmente, como resultado de las encuestas aplicadas a los docentes y los estudiantes, destacaron los datos mostrados en las Tablas 3 y 4 , respectivamente. 
Fortalecimiento de las competencias docentes para atender

el cyberbullying en una secundaria pública colombiana

Mauricio Alejandro Castro-Granados, Carmen MedinaAlmeida, Leonardo David Glasserman Morales

Tabla 3. Principales resultados de la encuesta a los docentes

\begin{tabular}{|c|c|}
\hline Pregunta & Resultado \\
\hline $\begin{array}{l}\text { ¿El cyberbullying es un conflicto presente en } \\
\text { esta institución educativa? }\end{array}$ & $\begin{array}{l}90 \% \text { reconoció la presencia del cyberbullying } \\
\text { entre estudiantes. }\end{array}$ \\
\hline $\begin{array}{l}\text { ¿La capacitación en cyberbullying es útil para } \\
\text { mejorar el ambiente escolar? }\end{array}$ & $\begin{array}{l}95 \% \text { consideró que la capacitación realizada } \\
\text { sobre el cyberbullying permitirá generar } \\
\text { propuestas a los directivos para atender este } \\
\text { problema. }\end{array}$ \\
\hline $\begin{array}{l}\text { ¿La capacitación recibida le permitió mejorar } \\
\text { su competencia docente para la atención de } \\
\text { conflictos escolares virtuales? }\end{array}$ & $\begin{array}{l}\text { 95\% reconoció la necesidad de recibir } \\
\text { capacitación constante en el tema de resolución } \\
\text { de conflictos. }\end{array}$ \\
\hline $\begin{array}{l}\text { ¿La capacitación en cyberbullying le permitió } \\
\text { reflexionar sobre su rol como mediador o } \\
\text { conciliador en conflictos escolares virtuales? }\end{array}$ & $\begin{array}{l}90 \% \text { reconoció que la capacitación que fue } \\
\text { parte de este proyecto, los hizo reflexionar } \\
\text { sobre su rol como mediadores y conciliadores } \\
\text { en el cyberbullying. }\end{array}$ \\
\hline $\begin{array}{l}\text { ¿Fortalecer las competencias docentes facilita } \\
\text { la atención y prevención del conflicto escolar } \\
\text { virtual entre los estudiantes de secundaria? }\end{array}$ & $\begin{array}{l}96 \% \text { consideró que la capacitación recibida } \\
\text { en Moodle permitirá mejorar la competencia } \\
\text { para el manejo, atención y prevención del } \\
\text { cyberbullying. }\end{array}$ \\
\hline $\begin{array}{l}\text { ¿Considera que los directivos de la institución } \\
\text { deben involucrarse más en la atención y } \\
\text { prevención del cyberbullying? }\end{array}$ & $\begin{array}{l}86 \% \text { señaló que la falta de capacitación } \\
\text { institucional les ha impedido mediar conflictos } \\
\text { escolares. }\end{array}$ \\
\hline
\end{tabular}

Fuente: Elaboración propia.

Cabe destacar, que en la encuesta aplicada a los estudiantes también se incluyeron algunas preguntas dicotómicas cuyas respuestas revelaron lo siguiente: $55 \%$ del total de estudiantes respondió que ha conocido casos de otros compañeros que han sido víctimas de cyberbullying. De igual manera, en la muestra encuestada, 19\% respondió que ha sido víctima de cyberbullying. Ahora bien, un poco más de la mitad de los estudiantes encuestados (56\%) afirmó que no confía en sus maestros para que manejen el conflicto. De igual manera, 53\% afirmó que sí sabe a quién recurrir en caso de ser víctima de acoso virtual (Tabla 4 ). 
Tabla 4. Principales resultados de la encuesta a los estudiantes

\begin{tabular}{|c|c|}
\hline Pregunta & Resultado \\
\hline $\begin{array}{l}\text { ¿Recurres a tus maestros en caso de sufrir } \\
\text { cyberbullying? }\end{array}$ & $\begin{array}{l}56 \% \text { afirmó no confiar en el docente para } \\
\text { solucionar un caso de cyberbullying. }\end{array}$ \\
\hline $\begin{array}{l}\text { ¿Consideras que tus maestros están preparados } \\
\text { para atender y manejar los conflictos de } \\
\text { cyberbullying? }\end{array}$ & $\begin{array}{l}77 \% \text { consideró que es necesaria la constante } \\
\text { capacitación del docente para ser un mediador } \\
\text { en casos de cyberbullying. }\end{array}$ \\
\hline $\begin{array}{l}\text { ¿Crees que los docentes de la institución son } \\
\text { indiferentes ante los conflictos de cyberbullying } \\
\text { que surgen entre los adolescentes? }\end{array}$ & $\begin{array}{l}34 \% \text { de los estudiantes respondió que } \\
\text { los docentes no son indiferentes ante los } \\
\text { conflictos de cyberbullying que surgen entre los } \\
\text { adolescentes. De igual manera, } 32 \% \text { no supo } \\
\text { responder ante esa afirmación (ni de acuerdo, ni } \\
\text { en desacuerdo). }\end{array}$ \\
\hline $\begin{array}{l}\text { ¿Consideras que los docentes deben saber } \\
\text { usar las Tecnologías de la Información y la } \\
\text { Comunicación, a fin de poder intervenir en } \\
\text { casos de cyberbullying que se presenten en } \\
\text { redes sociales, foros, chats, etcétera? }\end{array}$ & $\begin{array}{l}78 \% \text { reconoció la importancia de que los } \\
\text { docentes sepan usar las TIC para poder } \\
\text { intervenir en casos de cyberbullying que se } \\
\text { presenten a través de diferentes redes sociales, } \\
\text { foros o chats. }\end{array}$ \\
\hline $\begin{array}{l}\text { ¿Crees que los padres de familia deben } \\
\text { intervenir, junto con los maestros, para poner } \\
\text { fin a los problemas de cyberbullying que se } \\
\text { generan entre los alumnos? }\end{array}$ & $\begin{array}{l}90 \% \text { consideró que los padres también son } \\
\text { responsables y deben intervenir junto con los } \\
\text { maestros para poner fin al cyberbullying. }\end{array}$ \\
\hline $\begin{array}{l}\text { ¿Consideras que los directivos, deben intervenir } \\
\text { en los casos cyberbullying? }\end{array}$ & $\begin{array}{l}81 \% \text { señaló que los directivos deben intervenir } \\
\text { en esta problemática. }\end{array}$ \\
\hline $\begin{array}{l}\text { ¿En caso de padecer cyberbullying, tienes la } \\
\text { confianza para pedir ayuda a los directivos de } \\
\text { tu escuela? }\end{array}$ & $\begin{array}{l}\text { Solo } 50 \% \text { indicó tener confianza para recurrir } \\
\text { a los directivos en caso de ser víctima de } \\
\text { cyberbullying. }\end{array}$ \\
\hline $\begin{array}{l}\text { ¿Crees que en casos graves de cyberbullying, } \\
\text { se deberían aplicar sanciones de índole penal, } \\
\text { fuera de la escuela? }\end{array}$ & $\begin{array}{l}75 \% \text { consideró que deben aplicarse } \\
\text { sanciones de índole penal en casos graves de } \\
\text { cyberbullying. }\end{array}$ \\
\hline
\end{tabular}

Fuente: Elaboración propia.

Finalmente, Teddlie y Tashakkori (2009) afirman que las inferencias derivadas de la fase cualitativa y cuantitativa en el diseño mixto, deben presentar una consistencia interpretativa. Por lo tanto, para dar validez una de las estrategias para el análisis de los datos fue la triangulación a partir de los resultados cualitativos y cuantitativos, 
para poder realizar metainferencias derivadas de los instrumentos aplicados y así lograr consistencia en el estudio (Valenzuela \& Flores, 2012). Asimismo, se consideraron los criterios de credibilidad y dependencia cualitativas contemplados por Guba y Lincoln (1989).

\subsection{Análisis e interpretación}

En este apartado se realiza la discusión final de los datos recolectados en las dos fases de este estudio. Para ello, se interpretan todos los datos y se concluye con las metainferencias propias de los métodos mixtos (Hernández et al., 2010), con el fin de lograr un mayor entendimiento del fenómeno indagado (Johnson \& Onwuegbuzie, 2004).

Lo anterior, implica dar respuesta a la pregunta principal de investigación, mediante la triangulación de diversas fuentes, es decir, los resultados de las fases del estudio, con los fundamentos teóricos. Parafraseando dicha interrogante, se pretendió analizar cuáles eran las competencias docentes que debía fortalecer el profesorado para la atención del cyberbullying. Al respecto, los resultados obtenidos a través de las diferentes entrevistas evidenciaron que se debe desarrollar un proceso de diálogo y comunicación constante, conocer al estudiante, así como saber usar y estar adecuadamente familiarizado con los medios de comunicación (particularmente las redes sociales), además de ser objetivo y neutral ante los diferentes conflictos. En palabras de Cano (2005), se requiere que los docentes puedan fortalecer competencias que permitan establecer relaciones interpersonales satisfactorias para la resolución de conflictos, el uso de las TiC y la autoevaluación constante.

Con relación a los datos obtenidos en las encuestas, el profesorado manifestó interés por recibir una constante capacitación en la atención del cyberbullying, y los estudiantes confirmaron que sus maestros requieren capacitarse en esta problemática, al igual que en el uso de las Tic. De igual manera, la coordinadora reconoció la importancia de capacitar a los docentes para que funjan como verdaderos mediadores.

Por lo tanto, los resultados presentados coincidieron con lo expuesto por Zeus y Skiffington (2000), quienes expresan que las competencias interpersonales en el contexto social, deben desarrollarse para fortalecer el manejo de conflictos, la comunicación eficaz y la flexibilidad. Asimismo, Frola y Velásquez (2011) consideran que se requiere fortalecer la competencia comunicativa para desarrollar las comunicaciones 
o interacciones asertivas entre docente y educando, con el fin de poder mediar en los conflictos y lograr la solución de estos. Puede afirmarse que ello se traducirá en mayor confianza hacia los maestros y facilitará la prevención, mediación y manejo del cyberbullying. Todo lo anterior deberá verse respaldado por la presencia de todos los directivos del colegio.

En consecuencia, cada uno de los docentes debe propender a mejorar sus competencias interpersonales, laborales y profesionales; en particular, asumir el rol de mediadores, comunicadores y facilitadores para comprender las diferentes situaciones que influyen en los conflictos de acoso entre los estudiantes, y así poder establecer estrategias como equipo docente que realiza un trabajo colegiado para atender, manejar y prevenir los conflictos de cyberbullying. Sin embargo, es necesario precisar que todo eso se logrará con el fundamental apoyo directivo y de todo el personal administrativo. Asimismo, se debe fomentar en los estudiantes el respeto hacia otros individuos, en cuanto a las opiniones, las prácticas, las orientaciones y los gustos de los demás, con el fin de poder lograr procesos de comunicación e interacción apropiados entre pares, ya sean de forma presencial o por medio del uso de las Tic. Obviamente, es necesario fomentar los valores de manera permanente entre los alumnos. Ello también permitirá desarrollar en los estudiantes un rol participativo al discutir las acciones y estrategias apropiadas para prevenir el cyberbullying, además de establecer mejores normas para la convivencia y seguridad en el colegio, tal y como lo tipifica la Ley de Infancia y Adolescencia (Gobierno Nacional de Colombia, 2013).

\section{Conclusiones}

Por todo lo anterior, es necesario precisar que el docente debe comprender y asimilar la función moral y social que ejercerá en su labor, especialmente cuando en la actual sociedad del conocimiento los estudiantes son considerados nativos digitales (Prensky, 2001). Asimismo, es preciso que el profesorado visualice la formación del alumnado como un proceso integral, que comprenda el desarrollo de las dimensiones cognitiva, emocional y afectiva del ser humano, que son trascendentales para la vida (García \& Martínez, 2014).

Consecuentemente, sobre el objetivo específico donde se pretendió determinar las competencias que requieren fortalecer los docentes para el manejo del cyberbullying, destacaron las siguientes: la competencia comunicativa, acentuando el conocer 
las expectativas, intereses, sueños, inquietudes, problemas, condiciones familiares, económicas y sociales de cada estudiante. Las competencias interpersonales, para mejorar la interacción, relación y comunicación entre el docente y los estudiantes, al igual que entre los mismos maestros. Además, el profesorado reconoció la importancia y necesidad de seguir capacitándose para usar adecuadamente las TIC, como un posible escenario de aprendizaje y, obviamente, como un espacio donde también se generan esta clase de conflictos entre los estudiantes. Lo que implica la presencia y acompañamiento de docentes, administrativos y padres. Finalmente, se requiere fortalecer las competencias orientadas a la resolución de conflictos, para poder generar las estrategias que permitan manejar, atender y prevenir el cyberbullying.

En cuanto al objetivo relativo a las estrategias implementadas por los docentes y según lo señala Torres (2010), las estrategias señaladas por ellos no son diferentes a mediar en un conflicto presencial entre pares; es decir, los maestros asumen un rol pasivo ante los casos de cyberbullying y ellos mismos reconocen no estar capacitados para manejar los conflictos. No basta sólo actuar intuitivamente, o remitir los casos al área administrativa. Por lo tanto, es relevante enfatizar que los maestros reconocieron la importancia de asumir un rol activo y dinámico para la generación de estrategias orientadas a la intervención, prevención y mediación del cyberbullying entre estudiantes, además de reconocer que necesitan capacitarse para lograrlo eficazmente. En consecuencia, toman relevancia las aportaciones de Lozano (2005), quien considera que los docentes deben ser comunicadores, mediadores y asesores para lograr entender y trabajar con individuos que presentan diferentes condiciones, ya sean sociales, económicas, culturales y religiosas.

Sobre el curso impartido en Moodle y respecto al cumplimiento del cuarto objetivo, se pretendió iniciar la capacitación en diferentes aspectos, como saber qué es el cyberbullying, las causas que lo originan, tipos de agresiones en un contexto virtual, el perfil de los actores en este conflicto, al igual que las consecuencias que se desencadenan con esta clase de agresión. Es necesario precisar que los docentes manifestaron no estar capacitados para mediar, atender y prevenir los casos de cyberbullying entre estudiantes. Sin embargo, posterior a la primera capacitación que derivó de este estudio, consideraron que podrán diseñar estrategias para mediar esta clase de conflictos y afirmaron que necesitan asumir el rol de mediadores ante este problema, abandonando la actuación intuitiva. Asimismo, consideraron que las TIC son una gran herramienta y que requieren capacitación en el uso de las nuevas tecnologías, en especial las redes sociales, espacio donde se genera con mayor frecuencia el cyberbullying. En 
Fortalecimiento de las competencias docentes para atender

el cyberbullying en una secundaria pública colombiana

Mauricio Alejandro Castro-Granados, Carmen MedinaAlmeida, Leonardo David Glasserman Morales

este sentido, es pertinente enfatizar que todas las futuras capacitaciones del colegio deberán adaptarse al perfil y necesidades del profesorado, con una atención personalizada donde se consideren las peculiaridades de los casos de cyberbullying que se han presentado en la institución.

Por otra parte, los docentes consideraron necesaria la participación oportuna de los directivos del colegio ante este problema, a partir de capacitaciones constantes. Sobre su responsabilidad en cuanto a brindar capacitaciones al profesorado (quinto objetivo específico), ellos también enfatizaron la necesidad de participación y trabajo en equipo con padres de familia, entidades gubernamentales y privadas, para poder enfrentar esta situación. Además de lo anterior, es necesario enfatizar que deben fortalecerse los valores en los alumnos, a fin de reforzar lo que debe aprenderse desde el hogar, sin olvidar la evidente necesidad de fortalecer la confianza de los estudiantes hacia sus maestros; aspecto esencial que facilitará el diálogo, el manejo de conflictos y que los alumnos comuniquen a los docentes lo que están padeciendo. De gran relevancia es la necesidad de generar espacios de diálogo con los estudiantes para abordar el cyberbullying.

Finalmente, esta investigación cuyo alcance fue descriptivo, brinda la oportunidad de seguir indagando sobre el cyberbullying. Por ello se requieren futuros estudios cuyos objetivos permitan determinar la tasa de estudiantes que son víctimas, identificar los diferentes tipos de acoso que se presentan, determinar los medios digitales más utilizados en las agresiones, conocer el nivel de inteligencia emocional en víctimas y agresores de secundaria, además de analizar la perspectiva de las víctimas que han intentado suicidarse. Según Lucas et al. (2016), es necesario seguir indagando sobre el cyberbullying, especialmente en la correcta identificación de esta forma de acoso, a fin de garantizar su adecuada prevención e intervención.

Es necesario enfatizar que uno de los principales retos del docente es educar al estudiante como un ser social, con principios y valores (UNESCO, 2013). Por lo tanto, el profesorado debe reconocer que el cyberbullying es una problemática presente entre los estudiantes de cualquier institución educativa, que debe atenderse para evitar la generación de tendencias delictivas graves, que incluso podrían trascender hasta la vida adulta. 
Fortalecimiento de las competencias docentes para atender

el cyberbullying en una secundaria pública colombiana

Mauricio Alejandro Castro-Granados, Carmen MedinaAlmeida, Leonardo David Glasserman Morales

\section{Lista de Referencias}

Aftab, P. (2006). Cyberbullying. Guía práctica para madres, padres y personal docente. Bilbao: Fundación EDEX.

Aznar, I., Cáceres, M. P., \& Hinojo, F. (2007). Estudio de la violencia y conflictividad escolar en las aulas de educación primaria a través de un cuestionario de clima de clase: el caso de las provincias de Córdoba y Granada (España). Revista Electrónica Iberoamericana sobre Calidad, Eficacia y Cambio en Educación, 96(1), 164-177. Recuperado de http://www.rinace.net/arts/volsnumi/artg.htm

Blaya, C. (2010). Violencia y acoso escolar: Concepto, incidencia y factores de riesgo y protección. Valencia: Universidad Internacional Valenciana.

Borges, S., Bottino, C., Gómez, C., Lobo, A., \& Silva, W. (marzo, 2015). Cyberbullying and adolescent mental health: Systematic review. Cuadernos de Saúde Pública, 31(3), 463-475. Recuperado de http://www.scielo.br/scielo.php?pid=So102311 X2015000300463\&script $=$ sci_arttext

Caba de la, M. A., \& López, R. (septiembre-diciembre, 2013). La agresión entre iguales en la era digital: estrategias de afrontamiento de los estudiantes de último ciclo de Primaria y del primero de Secundaria. Revista de Educación, 362, 247272. doi: 10-4438/1988-592X-RE-2011-362-160

Cano, E. (2005). Cómo mejorar las competencias de los docentes. Guía para la autoevaluación y el desarrollo de las competencias del profesorado. Barcelona: Graó.

Cowie, H. (abril, 2013). El impacto emocional y las consecuencias del ciberacoso. Revista Digital de la Asociación Convives, 3, 16-24. Recuperado de http://convivesenlaescuela.blogspot.mx/2015/o2/resumen-despues-de-analizar-el-contexto.html

Creswell, J. W. (2009). Research Design: Qualitative, quantitative, and mixed methods approaches. Londres: Sage.

Echeverría, B. (junio, 2002). Gestión de las competencias de acción profesional. Revista de Investigación Educativa, 2o(1), 7-43. Recuperado de http://revistas. um.es/rie/article/view/97411

Frade, L. (2009). Desarrollo de las competencias en educación: desde preescolar hasta bachillerato. México: Inteligencia educativa.

Frola, P., \& Velásquez, J. (2011). Desarrollo de las competencias docentes a partir de trayectos formativos. México: Centro de Investigación Educativa y Capacitación Institucional.

Garaigordobil, M. (2011). Prevalencia y consecuencias del cyberbullying: una revi- 
Fortalecimiento de las competencias docentes para atender

el cyberbullying en una secundaria pública colombiana

Mauricio Alejandro Castro-Granados, Carmen MedinaAlmeida, Leonardo David Glasserman Morales

sión. International Journal of Psychology and Psychlogical Therapy, 11(2), 233254. Recuperado de http://www.ijpsy.com/volumen11/num2/295.html

García, A., \& Martínez, L. (agosto, 2014). El constructo "competencias" en docentes de Bachillerato. Entreciencias: Diálogos de la Sociedad del Conocimiento, 2(4), 163-170. Recuperado de https://doi.org/10.21933/J.EDSC.2014.04.070

García, G., Joffre, V., Martínez, G., \& Llanes, A. (enero, 2011). Cyberbullying: forma virtual de intimidación escolar. Revista Colombiana de Psiquiatría, 4o(1), 115130. Recuperado de http://www.scielo.org.co/pdf/rcp/v4on1/v4on1a10.pdf

Giménez, A. M. (2015). Estrategias de afrontamiento ante el cyberbullying. Una mirada cualitativa desde la perspectiva de los escolares. Campo abierto. Revista de Educación, 49-65. Recuperado de http://mascvuex.unex.es/revistas/index. $\mathrm{php} /$ campoabierto/article/view/1685

Gobierno Nacional de Colombia. (2013). Ley 1620 de 2013. Ley Nacional de Convivencia Escolar y Formación para el Ejercicio de los Derechos Humanos, la Educación para la Sexualidad y la Prevención y Mitigación de la Violencia Escolar. Recuperado de http://www.mineducacion.gov.co/1759/articles-327397_archivo pdf_proyecto decreto.pdf

Grasa, R. (1991). Resolución de conflictos. En M. Martínez \& J. Puig (Comps.), La educación moral. Perspectivas de futuro y técnicas de trabajo (pp. 105-102). Barcelona: Graó.

Guba, E. G., \& Lincoln, Y. (1989). Fourth generation evaluation. California: Sage.

Hernández, M. A. (2004). Los conflictos escolares desde la perspectiva familiar (Tesis doctoral inédita). Universidad de Murcia, España.

Hernández, R., Fernández, C., \& Baptista, P. (2010). Metodología de la Investigación. México: Mc Graw-Hill.

Henríquez-Ritchie, P., \& Organista, J. (2010). Clasificación de niveles de uso tecnológico: una propuesta con estudiantes de recién ingreso a la universidad. CPU-E, Revista de Investigación Educativa, 11, 85-109. Recuperado de http://revistas. uv.mx/index.php/cpue/article/view/61

Instituto Nacional de Tecnologías de la Comunicación. (2009). Guía legal sobre cyberbullying y grooming. Recuperado de https://www.educacion.navarra.es/documents/57308/57740/ciberbullyng.pdf/1c169fb5-b8ab-478f-b7f4-7e3d22adab14

Jiménez, Y., González, M., A., \& Hernández, J. (julio-diciembre, 2011). Propuesta de un modelo para la evaluación integral del proceso enseñanza-aprendizaje acorde con la Educación Basada en Competencias. CPU-E, Revista de Investi- 
Fortalecimiento de las competencias docentes para atender

el cyberbullying en una secundaria pública colombiana

Mauricio Alejandro Castro-Granados, Carmen MedinaAlmeida, Leonardo David Glasserman Morales

gación Educativa, 13, 34-58. Recuperado de http://revistas.uv.mx/index.php/ cpue/article/view/37

Johnson, R. B., \& Onwuegbuzie, A. J. (octubre, 2004). Mixed methods research: A research paradigm whose time has come. Educational Researcher, 33(7), 14-26. Recuperado de http://www.tc.umn.edu/ dillon/CI\%208148\%20Qual\%20 Research/Session\%2014/Johnson\%20\&\%20Onwuegbuzie\%2oPDF.pdf

Kowalski, R., Limber, S., \& Agatston, P. (2010). Cyberbullying: el acoso escolar en la era digital. Bilbao, España: Desclée de Brower.

Lozano, A. (2005). El éxito de la enseñanza. Aspectos didácticos de la faceta del profesor. Recuperado de https://www.etrillas.com.mx/ebookdetalle.php?isbn=97860 71713599\&estilo $=13$ \& tema $=0$ \#

Lucas, B., Pérez de Albéniz, A., \& Giménez, M. (2016). La evaluación del cyberbullying: situación actual y retos futuros. Papeles del Psicólogo, 37(1), 27-35. Recuperado de http://www.papelesdelpsicologo.es/vernumero.asp? ID=2659

Luengo, J. A. (2011). Cyberbullying. Guía de recursos para centros educativos en casos de ciberacoso. Madrid: Defensor del Menor en la Comunidad de Madrid.

Lukas, J. F., \& Santiago, K. (2004). Evaluación de centros escolares de educación secundaria del País Vasco. Revista Electrónica de Investigación Educativa, 6(2), 57-63. Recuperado de http://redie.uabc.mx/redie/article/view/101

Merriam, S. B. (2009). Qualitative research. A guide to design and implementation. San Francisco: Jossey-Bass.

Ministerio de Educación Nacional. (2009). Encuesta Nacional de Deserción Escolar (ENDE). Recuperado de http://www.mineducacion.gov.co/1621/articles-293664_archivo pdf resultados ETC.pdf

Moore, C. (1995). El proceso de mediación. Métodos prácticos para la resolución de conflictos. Barcelona: Granica.

Morales, T., Serrano, M. C., Miranda, D. A., \& Santos, A. (2014). Cyberbullying, acoso cibernético y delitos invisibles. Experiencias psicopedagógicas. México: Universidad Autónoma del Estado de México.

Navés, F. (enero-junio, 2015). Las TIC como recurso didáctico: ¿competencias o posición subjetiva? CPU-E, Revista de Investigación Educativa, 20, 238-248. Recuperado de http://revistas.uv.mx/index.php/cpue/article/view/1308

Olweus, D. (2001). Peer harassment in school. The plight of the vulnerable and victimized. Nueva York: The Guilford Press.

Oyhanarte, M. (1996). Los nuevos paradigmas y la mediación. En J. Gottheil \& A. Schi- 
ffrin (Coords.), Mediación: una transformación en la cultura (pp. 17-36). Buenos Aires: Paidós Ibérica.

Pantoja, A. (2005). La gestión de conflictos en el aula. Factores determinantes y propuestas de intervención. Madrid: Ministerio de Educación y Ciencia de España.

Piñuel, I. (2002). El acosador es una persona poco brillante. Madrid: Cegal.

Prensky, M. (2001). Nativos e inmigrantes digitales. Recuperado de http://www.marcprensky.com/writing/Prensky-NATIVOS\%20E\%20INMIGRANTES\%20DIGITALES\%20(SEK).pdf

Prieto, M., Carrillo, J., \& Lucio, L. (mayo-agosto, 2015). Violencia virtual y acoso escolar entre estudiantes universitarios: el lado oscuro de las redes sociales. Innovación Educativa, 15(68), 33-47. Recuperado de http://www.scielo.org.mx/scielo. php?script $=$ sci arttext\&pid $=S_{1665-26732015000200004 \& l n g=e s \& t \operatorname{lng}=e s}$

Puig, J. M. (abril, 1997). Conflictos escolares: una oportunidad. Cuadernos de Pedagogía, 257, 58-65. Recuperado de https://bo6elkarbizitzamintegia.files.wordpress.com/2010/05/unaoportunidad.pdf

Teddlie, C., \& Tashakkori, A. (2009). Foundations of mixed methods research. Integrating quantitative and qualitative approaches in the social and behavioral sciences. California: Sage Publications.

Tobón, S. (2005). Formación basada en competencias. Pensamiento complejo, diseño curricular y didáctica. Bogotá: Ecoe.

Todd, Z., Nerlich, B., \& McKeown, S. (2004). Introduction. En Z. Todd, B. Nerlich, S. McKeown \& D. Clarke (Eds.), Mixing methods in psychology (pp. 3-16). Inglaterra: Psychology Press.

Torres, N. Y. (enero-junio, 2010). Alternativas de solución frente a la violencia escolar. Zona Próxima, 12, 242-249. Recuperado de http://rcientificas.uninorte.edu. co/index.php/zona/article/viewArticle/1152/4685

Organización de las Naciones Unidas para la Educación, la Ciencia y la Cultura. (2013). Día Mundial de los Docentes 2013: ¡Un llamamiento a la docencia! Recuperado de http://unesdoc.unesco.org/images/o022/002235/223537s.pdf

Vaello, J. (2003). Resolución de conflictos en el aula. Madrid: Santillana.

Valenzuela, J. R., \& Flores, M. (2012). Fundamentos de investigación educativa. Monterrey: Editorial Digital del Tecnológico de Monterrey.

Villa, A., \& Poblete, M. (2007). Aprendizaje basado en competencias. Una propuesta para la evaluación de propuestas genéricas. Bilbao: Mensajero.

Zeus, P., \& Skiffington, S. (200o). Tutoría o coaching en el trabajo. Madrid: McGraw-Hill. 